ScIDoC

\section{Use of Intermediate Restorative Material among Clinical Dental Practitioners - A Questionnaire Based Survey}

Research Article

R.Kushali ${ }^{1}$, Jayalakshmi Somasundaram ${ }^{2 *}$, R.V.Geetha ${ }^{3}$

${ }^{1}$ Undergraduate Student, Saveetha Dental College and Hospitals, Saveetha Institute of Medical and Technical Sciences, Saveetha University, Chennai, India.

${ }^{2}$ Chief Scientist, White Lab- Material Research Centre, Saveetha Dental College and Hospitals, Saveetha Institute of Medical and Technical Sciences, Saveetha University, Chennai, 600077, India.

${ }^{3}$ Department of Microbiology, Saveetha Dental College and Hospitals, Saveetha Institute of Medical and Technical Sciences, Saveetha University, Chennai, 600077, India.

\title{
Abstract
}

The aim of this study is to assess the use of intermediate restorative material among clinical dental students and practitioners. Intermediate restorative material(IRM) is a polymer-reinforced Zinc oxide Eugenol composition restorative material designed for intermediate restorations intended to remain in place for no longer than one year. It may also be used as a base under restorative material and cements that do not resin components. The study was distributed to 200 practitioners who are professionally dentists, both practitioners and dental students. A questionnaire survey was prepared and circulated online which included 12 questions. The results revealed that around $95 \%$ of the study population were aware about the use of IRM and $5 \%$ unaware. Despite the limitations, our findings provide valuable information about the usage of intermediate restorative material among dental clinicians.

Keywords: Awareness; Clinical Practice; Intermediate Restorative Material; Properties.

\section{Introduction}

Temporary restorative materials are used for restoring the tooth temporarily until permanent restoration can be given. It covers the prepared part of the tooth structure, in order to maintain the occlusalspacel as the contact points as well [18]. It also provides insulation of the pulpal tissues and maintains the periodontal relationship. Sometimes in order to prepare indirect restorations such as inlays and onlays, permanent restoration cannot be preferred after tooth preparation [22]. Temporary restorations are also used for dental caries stabilization methods where many restorations are needed, and the problem can become worse even before it can be fully treated. Hence the temporary restorations are placed in order to stop caries progression. Temporary restoration can last approximately for one month and sometimes more. Bacterial infection has been declared as the most common cause of the pulpal and periradicular diseases. Therefore; the major goals of root canal treatment are the chemo mechanical debridement and sealing of the root canal system to eliminate the irritants [28]. Temporary restorations are commonly used to seal endodontic access cavities between patient visits and after completion of endodontic therapy to prevent coronal microleakage.

The ideal requirements of a temporary filling material are:

- It Should be easily removed from the cavity,

- It Should have a sedative effect to the tooth and promote pulp healing

- Reasonable strength and abrasive resistance, radiopaque.

- Reasonable setting time and has low flow after setting,

- Posses antibacterial property and marginal integrity

- low water sorption and solubility [20, 25].

Intermediate restorative material is designed for intermediate restorations intended to remain in place for up to one year [4]. The

*Corresponding Author

Jayalakshmi Somasundaram,

Chief Scientist, White Lab- Material Research Centre, Saveetha Dental College and Hospitals, Saveetha Institute of Medical and Technical Sciences, Saveetha University, Chennai, 600077, India.

Tel: 9360293308

E-mail: jayalakshmisomasundaram@saveetha.com

Received: August 10, 2019

Accepted: August 28, 2019

Published: August 30,2019

Citation: R.Kushali, Jayalakshmi Somasundaram, R.V.Geetha. Use of Intermediate Restorative Material among Clinical Dental Practitioners - A Questionnaire Based Survey. Int J Dentistry Oral Sci. 2019;S4:02:009:42-48. doi: http://dx.doi.org/10.19070/2377-8075-SI02-04009

Copyright: Jayalakshmi Somasundaram ${ }^{\circ} 2019$. This is an open-access article distributed under the terms of the Creative Commons Attribution License, which permits unrestricted use, distribution and reproduction in any medium, provided the original author and source are credited. 
liquid contains eugenol and the powder contains methyl acrylate resin in the form of dust. It is preferred for class 1 and class II cavities. IRM is composed of Zinc oxide interspersed in a matrix of organic material. The material powder consists of Zinc oxide, poly-methyl acrylate (PMMA) powder and pigments. The IRM material liquid consists of Eugenol, Acetic acid. Components and manipulated material are irritating to the eye and skin as well. It is necessary to avoid prolonged or repeated exposure of IRM with skin, oral soft tissue, and eyes [12]. Skin rashes, oral mucosa irritation, or other allergic reactions may result in susceptibility individuals [29]. Also, prolonged inhalation should be avoided. Before using the product, eye protection is to be used by both the patient and the clinician [15]. IRM material liquid bottles contain excess liquid for the amount of powder in 1 powder bottle. Providing excess allows some for natural liquid evaporation over opening repeatedly [13]. IRM should not be used in conjugation with resin-based adhesives, varnishes or restorations because eugenol may interfere with the hardening or can soften the polymeric components [27]. IRM should be mixed in equal proportion i.e., 1 level scoop to 1 drop of liquid, 6:1 by weight. This should be followed by homogeneous and streak free prior to application $[2,14]$. The current study is done to know the knowledge and the usage of IRM among clinical students and dental practitioners.

\section{Materials and Method}

A cross-sectional survey research approach using electronic distribution of a questionnaire was done and the sample size included 200 participants. The sampling method used in this study is stratified random survey sampling. To minimise sampling bias certain measures were taken which included framing straight forward questions that sounded simple and understandable. They were kept short and easy. A self structured questionnaire of 12 questions was prepared which were checked for validity by three internal experts from Saveetha dental College and also by three external experts outside Saveetha dental College. The questionnaire enquired about the awareness and usage of intermediate restorative material among the students of various dental colleges and dental practitioners in and around Chennai. This was the best approach for the collection of the data as a large number of dentists and clinical students were involved.The survey included 12 questions and was distributed to dental professionals of various dental colleges and hospitals.

\section{Results and Discussion}

In figure 1 profession of the study populationis discussed in which $64 \%$ of dental students of various colleges who were either BDS or MDS qualified in and around Chennai and $36 \%$ of them are dental practitioners who were either BDS or MDS qualified. In figure 2 Qualification of the study population is shown. It represents the qualification of the study population described in figure 1 . Out of which $56 \%$ of the participants were BDS qualified and $44 \%$ are MDS qualified. This includes the qualification of both, the students and the dental practitioners. In figure 3 , awareness of IRM is discussed. $95 \%$ of the respondents were aware about IRM and also use them in their clinical practice according to the need while on the other hand, a small percentage of the population, about $5 \%$ of them were unaware about it and

Figure 1. Depicts a pie chart showing the responses to the question " profession of the study population" which included $64 \%$ of dental students of various colleges who were either BDS or MDS qualified (blue) and 36\% of them are dental practitioners which includes both BDS or MDS qualified( red).

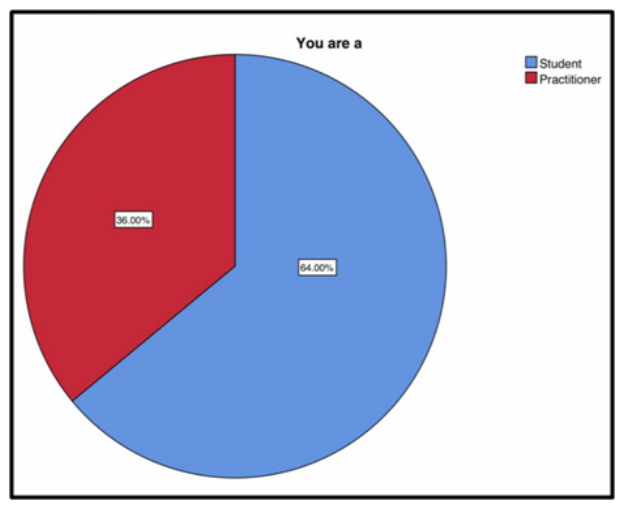

Figure 2. Depicts a pie chart showing the responses to the question "Qualification of the study population". Out of which $56 \%$ of the participants were BDS qualified which included both UG students and UG practitioners (blue) and $44 \%$ are MDS qualified including both.This includes the qualification of both, the PG students and PG practitioners.( red).

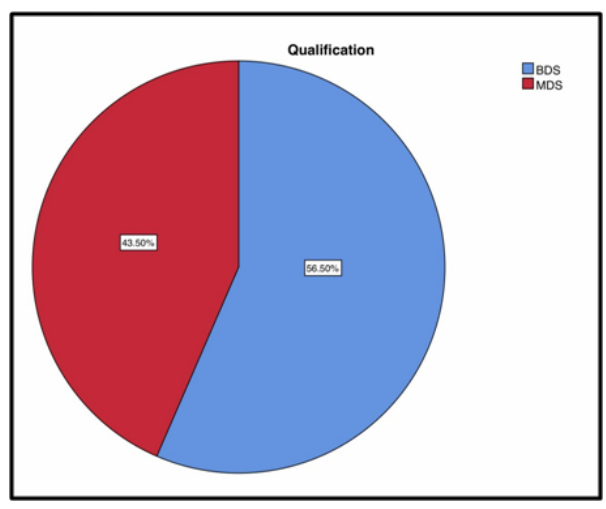


also do not use them in clinical practices and this could be due to the fact that IRM is a recent advancement [8].

In figure 4, Usage of IRM on various conditions IRM, being a temporary restorative material can be used in various procedures and treatment. $7 \%$ of the study population uses IRM as a base and a restorative material, $15 \%$ of them use it in the oral cavity undergoing multiple procedures [10]. On the other hand a majority of $78 \%$ of individuals use it in both the above conditions (green). It is a biocompatible strong cement since it is zinc oxide eugenol based [19]. Temporary restorative material has been developed to be applied to the dentine prior to the placement of the restorative material [21]. IRM may also be used as a base under cements and restorative materials that do not contain resin components, such as amalgams, and inlays and onlays. In figure 5, durability of IRM is discussed. $12 \%$ of the study population say that IRM stays in the oral cavity on average for about 0 to 6 months while $84 \%$ the participants agreed that it stays for an average of six months to one year in the oral cavity and $4 \%$ of them say it stays for more than a year. Studies reveal that IRM stays in place for an average of one year and not more [3].

In Figure 6, IRM is a modification of which material is discussed. $93 \%$ of the study population agree that IRM is a modification of zinc oxide Eugenol but a small population of $7 \%$ people believe that IRM is a modification of Glass Ionomer Cement which is actually incorrect.The modification of Zinc oxide Eugenol is done to overcome the disadvantages of $\mathrm{ZOE}$ which are low in strength and production of inflammation. The other modification of ZOE is EBA [8]. In Figure 7 Usage of IRM is discussed. In the pie graph $89 \%$ of the study population agree that IRM is used for class I and class II restoration, $7 \%$ of them say it is used for class IIIand class IV restorations while a small group of $4 \%$ of the people believe that is used for class five restoration [16]. In Figure 8,Properties of IRM are discussed. $6 \%$ of the study population agree that IRM has a sedative like property or hypersensitive pulp,

Figure 3. Depicts a pie chart showing the responses to the question "Awareness on IRM" 95\% of the respondents were aware about IRM (blue ) and also used them in their clinical practice according to the need while on the other hand, a small percentage of the population, about $5 \%$ of them were unaware( red).

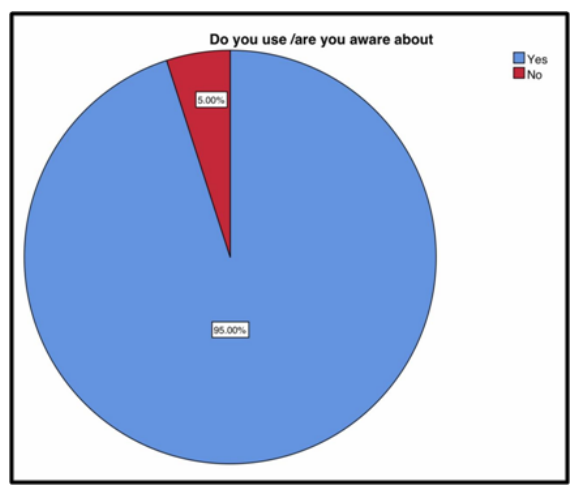

Figure 4. Depicts a pie chart showing the responses to the question "Usage of IRM on various conditions" $7 \%$ of the study population uses IRM as a base and a restorative material (blue ), 15\% of them use it in the oral cavity undergoing multiple procedures( red). On the other hand a majority of $78 \%$ of individuals use it in both the above conditions (green).

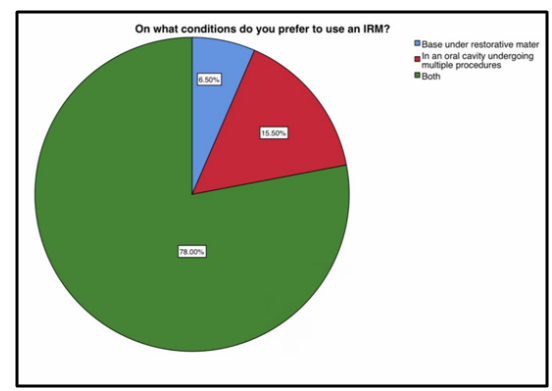

Figure 5. A pie chart showing the responses to the question "How long IRM stays on average" $12 \%$ of the study population say that IRM stays in the oral cavity on average for about 0 to 6 months (blue) while $84 \%$ the participants agreed that it stays for an average of six months to one year in the oral cavity ( red ) and $4 \%$ of them say it stays for more than a year (green).

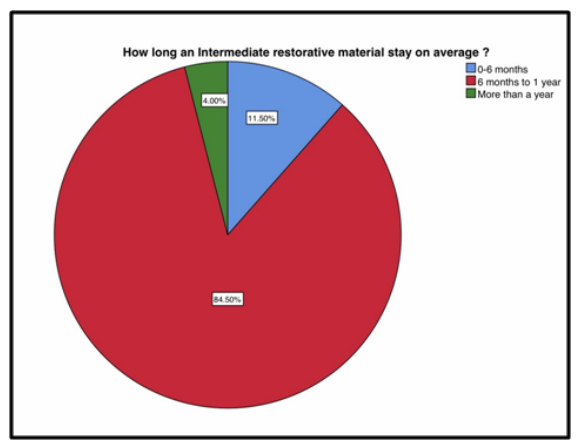


Figure 6. A pie chart showing the responses to the question "IRM is a modification of which material" $93 \%$ of the study population agree that IRM is a modification of zinc oxide Eugenol (blue) but a small population of 7\% people believe that IRM is a modification of Glass Ionomer Cement which is actually incorrect (red).

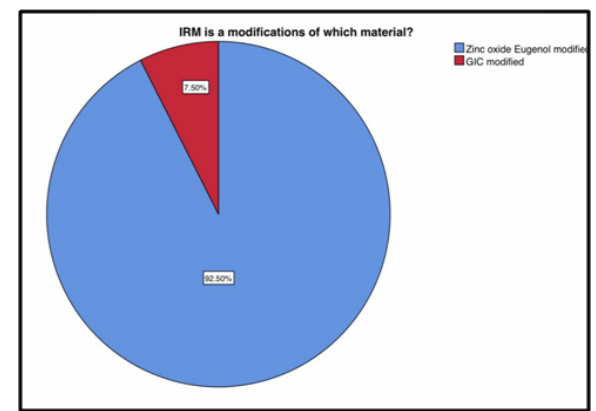

Figure 7. A pie chart showing the responses to the question "Usage of IRM" $89 \%$ of the study population agree that IRM is used for class I and class II restoration( blue ), 7\% of them say it is used for class IIIand class IV restorations (red)while a small group of $4 \%$ of the people believe that is used for class five restoration (green).

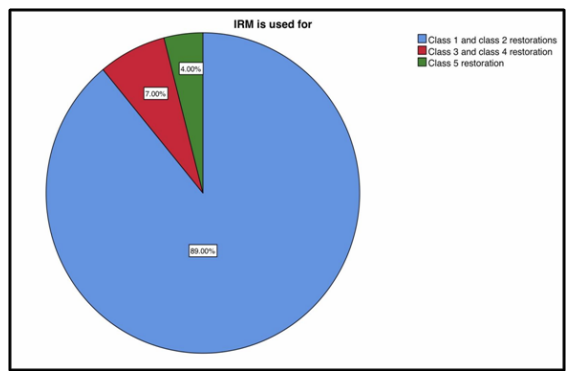

Figure 8. A pie chart showing the responses to the question "Properties of IRM." $6 \%$ of the study population agree that IRM has a sedative like property or hypersensitive pulp (blue), $5 \%$ of them agree that it has thermal insulator property( red ) and 3\% of them believe that it has low solubility property( green), $4 \%$ of them say it has excellent abrasion resistance(orange) while a majority of $82 \%$ of them agree that it has all the above properties. IRM has improved compressive strength, operation, assistance and hardness (yellow).

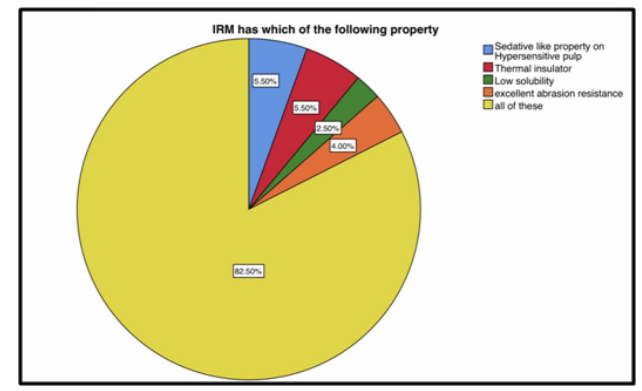

Figure 9. A pie chart showing the responses to the question "Contraindications of IRM", 5\% of the study population say IRM is contraindicated in case of patients who are allergic to use Eugenol reactions(blue), $2 \%$ of them believe that it shouldn't be used to patients who have allergy to words acrylate resins(red), $8 \%$ of them feel that direct application of IRM on dental pulp(green), while a majority of $86 \%$ of them agree that it is not advisable to use IRM in all of the above given conditions since adverse reaction may occur(orange).

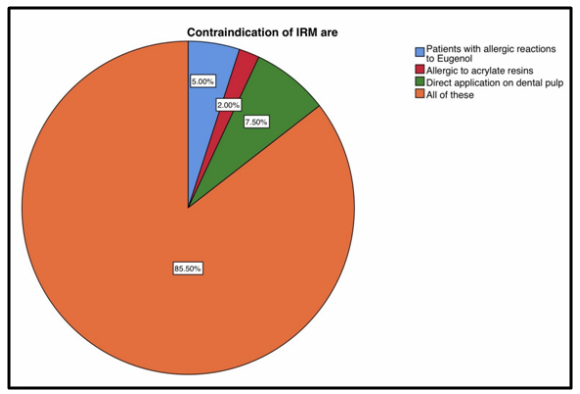

$5 \%$ of them agree that it has thermal insulator property and $3 \%$ of them believe that it has low solubility property, $4 \%$ of them say it has excellent abrasion resistance while a majority of $82 \%$ of them agree that it has all the above properties. IRM has improved compressive strength, operation, assistance and hardness [6]. The eugenol present in the polymer- reinforced zinc oxide- eugenol composition gives the material a lot of properties and few of them are sedative-like qualities on hypersensitive tooth pulp and can work as a good thermal insulator [31]. Figure 9 discusses about the Contraindications of IRM. 5\% of the study population say IRM is contraindicated in case of patients who are allergic to use Eugenol reactions, 2\% of them believe that it shouldn't be 
Figure 10. A pie chart showing the responses to the question "Usage of IRM in case of resin based restorations", $86 \%$ of the respondents disagree that the use of IRM in case of future treatment of resin based restorations(red). On the other hand , $14 \%$ of them think it is advisable to do so (blue).

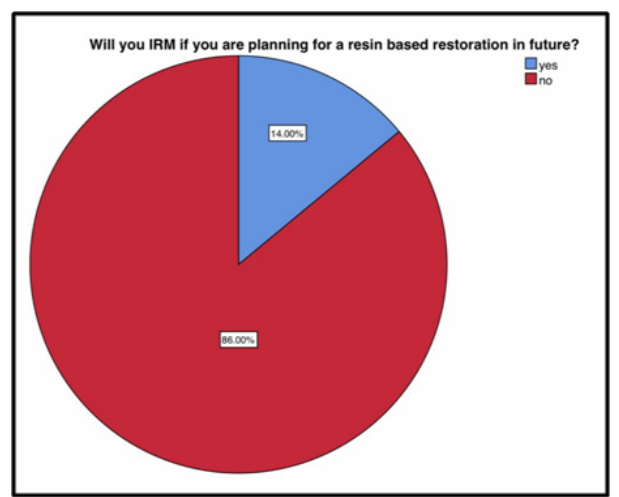

Figure 11. This bar graph represents correlation of qualification and profession. $\mathrm{X}$ axis represents qualification and $\mathrm{Y}$ axis represents profession. The blue color denotes students and red denotes practitioners. The graph shows that out of $64 \%$ of the students , $49 \%$ are BDS and $15 \%$ are MDS. While out $36 \%$ of the practitioners $7.5 \%$ are BDS qualified and $28.5 \%$ are MSD qualified. $P$ value $=0.000$, statistically significant( Chi square test). Among the qualifications , BDS( $49 \%)$ are students.

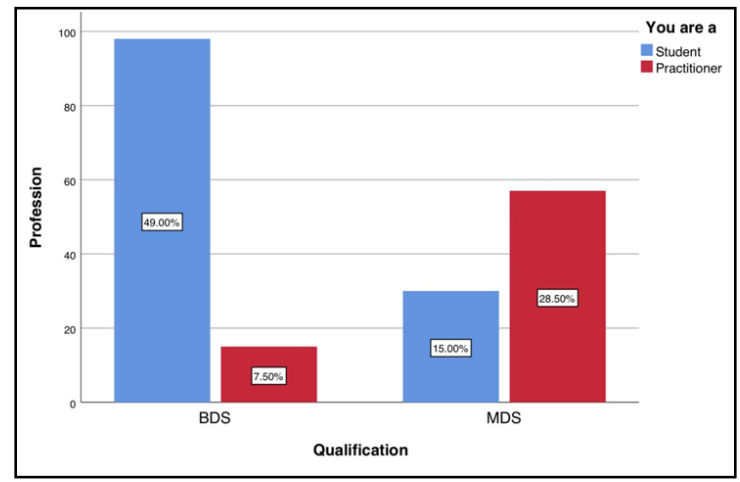

Figure 12. This graph represents the correlation of qualification and durability of the restoration. $\mathrm{X}$ axis represents qualification and $\mathrm{Y}$ axis represents durability of the restoration where blue color denotes 0-6 months, red denotes 6 months to 1 year and green color denotes more than a year.The graph shows that out of $11.5 \%$ who responded 0-6 months , they were all BDS qualified. Out of 84.5 of the respondents who chose 6 months to 1 year, $41 \%$ were BDS qualified and $43.5 \%$ are MSD qualified and $4 \%$ of the respondents who chose more than 1 year were BDS qualified. $P$ value $=0.000$, statistically significant( Chi square test). Among the qualifications, MDS( 43.5\%) responded more for 6 months to 1 year to be the average stay of IRM.

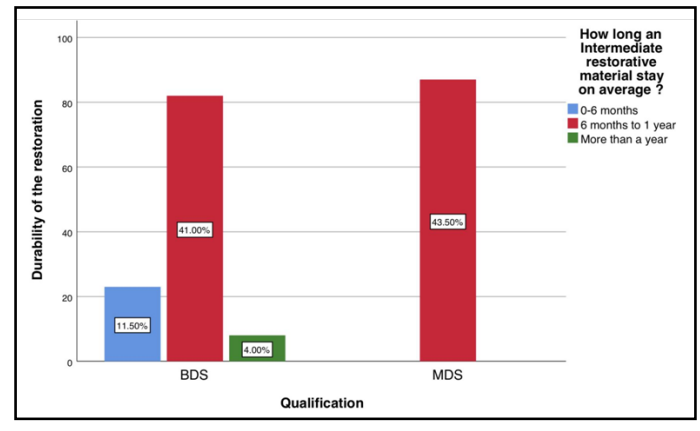

used to patients who have allergy to words acrylate resins, $8 \%$ of them feel that direct application of IRM on dental pulp, while a majority of $86 \%$ of them agree that it is not advisable to use IRM in all of the above given conditions since adverse reaction may occur [17]. IRM is contraindicated for use in patients who have a known hypersensitivity or severe allergic reaction to eugenol, acrylate resin or any of the components [26]. It is contraindicated for direct application to dental pulp tissue(direct pulp capping) [30] and as base under resin containing adhesives, restoratives or cements because eugenol may interfere with the hardening and/ or cause softening of the polymeric components [24]. In Figure
10 Usage of IRM in case of resin based restorations, $86 \%$ of the respondents disagree that the use of IRM in case of future treatment of resin based restorations. On the other hand, 14\% of them think it is advisable to do so. Study reveals that IRM to be used as a base on the non-resin restoration. It is also known that Zinc oxide Eugenol inhibits polymerisation of composites [5].

Numerous studies are done on Intermediate restorative material. A study by Hussainy et al., was done on investigating materials which included prototype-radiopacifiedtricalcium silicate cement, biodentine, bioaggregate and Intermediate Restorative 
Figure 13. This graph represents the correlation of qualification and IRM is a modification of which material. $X$ axis represents qualification and $Y$ axis represents IRM is a modification of which material where blue color denotes Zinc oxide eugenol modified and red denotes GIC modified .The graph shows that out of $92.5 \%$ of the respondents who chose ZOE modified , $49 \%$ were BDS and $43.5 \%$ were MDS. While out $7.5 \%$ of the respondents who chose GIC modified were BDS qualified. $P$ value $=0.000$, statistically significant( Chi square test). Among the qualifications, BDS( $49 \%)$ chose IRM to be the modification of ZOE.

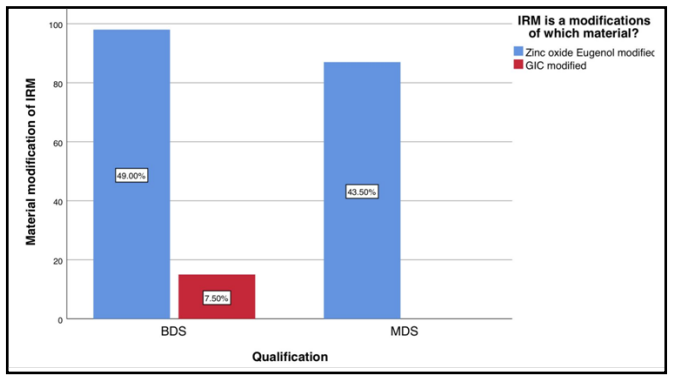

Figure 14. This graph represents the correlation of qualification and usage of IRM in case of future resin based restorations. $\mathrm{X}$ axis represents qualification and $\mathrm{Y}$ axis represents usage of IRM in case of resin based restoration where blue color denotes yes and red denotes no. The graph shows that $14 \%$ of the participants who told yes , $12 \%$ were BDS and $2 \%$ were MDS. While out $86 \%$ of the participants who told no , $44.5 \%$ were BDS qualified and $41.5 \%$ were MSD qualified. $P$ value $=0.001$, statistically significant (Chi square test). Among the qualifications, BDS(44.5\%) chose no for IRM in case of future resin based restorations.

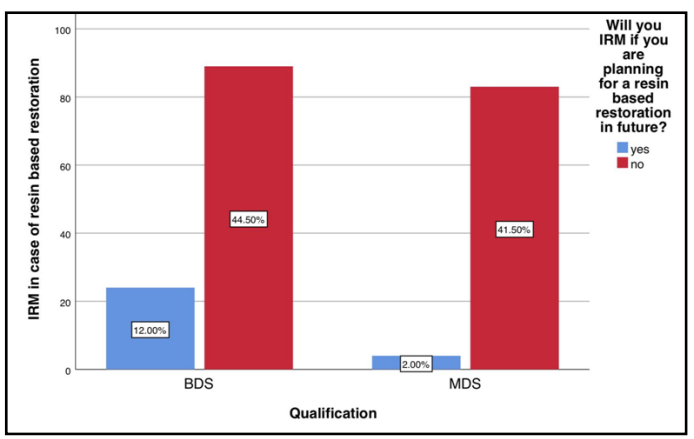

Material (IRM) which is of interest. The $\mathrm{pH}$ and the calcium ion concentration of the leachate were investigated [7]. The radiopacity of intermediate restorative materials should be sufficient to enable the clinician to distinguish the material from normal and decalcified tooth structure [11]. Studies have also been done to determine the relative radiopacities of intermediate restorative materials, including a newly introduced high-viscosity, self-cured, condensable glass ionomer material.periapical tissue response of 4 different retrograde root-filling materials, ie, intermediate restorative material, thermoplasticized gutta-percha, reinforced zinc oxide cement (Super-EBA), and mineral trioxide aggregate (MTA), in conjunction with an ultrasonic root-end preparation technique in an animal model. When a study was carried out to evaluate and compare the sealing properties, water absorption and solubility of IRM (intermediate restorative material), Cavit $G$ and GC Caviton it was observed that GC Caviton showed least microleakage and least water absorption followed by IRM and Cavit $\mathrm{G}[1,23]$.

\section{Conclusion}

The above study reveals that the majority of the study population were aware about IRM and its appropriate use. It reveals that the practitioners are well aware about the contraindications, precaution, ratio of water and powder, use in appropriate cavities, average stay in a place and also properties of intermediate restorative material.

\section{Acknowledgement}

The authors are thankful to Saveetha Dental College for providing a platform to express our knowledge.

\section{References}

[1]. Baskran RN, Pradeep S. Recent advancement of local anasthesia advancement to recent advancement of local anaesthesia administration. Research Journal of Pharmacy and Technology. 2016;9(10):1761-4.

[2]. Bhaskar SN, Cutright DE, Beasley JD, Boyers RC. Pulpal response to four restorative materials. Oral Surgery, Oral Medicine, Oral Pathology. 1969 Jul 1;28(1):126-33.

[3]. DuBois DJ, Reichl RB, Hondrum SO. The comparative radiopacity of Fuji IX-GP, an intermediate restorative material. Military medicine. $2000 \mathrm{Apr}$ $1 ; 165(4): 278-82$.

[4]. Fischer EJ, Arens DE, Miller CH. Bacterial leakage of mineral trioxide aggregate as compared with zinc-free amalgam, intermediate restorative material, and Super-EBA as a root-end filling material. Journal of endodontics. 1998 Mar 1;24(3):176-9.

[5]. Gundam S, Patil J, Venigalla BS, Yadanaparti S, Maddu R, Gurram SR Comparison of marginal adaptation of mineral trioxide aggregate, glass ionomer cement and intermediate restorative material as root-end filling materials, using scanning electron microscope: An in vitro study. J Conserv Dent. 2014 Nov;17(6):566-70. PubmedPMID: 25506146.

[6]. Hadis MA, Shortall AC, Palin WM. Competitive light absorbers in photoactive dental resin-based materials. Dent Mater. 2012 Aug;28(8):831-41. Pubmed PMID: 22578661.

[7]. Hussainy SN, Nasim I, Thomas T, Ranjan M. Clinical performance of resinmodified glass ionomer cement, flowable composite, and polyacid-modified resin composite in noncarious cervical lesions: One-year follow-up. Journal of conservative dentistry: JCD. 2018 Sep;21(5):510.

[8]. Janani K, Ajitha P, Sandhya R. Improved quality of life in patients with 
dentin hypersensitivity. Saudi Endodontic Journal. 2020 Jan 1;10(1):81-.

[9]. Janani K, Palanivelu A, Sandhya R. Diagnostic accuracy of dental pulse oximeter with customized sensor holder, thermal test and electric pulp test for the evaluation of pulp vitality: an in vivo study. Brazilian Dental Science. 2020 Jan 31;23(1):8-p.

[10]. Jose J, Subbaiyan H. Different Treatment Modalities followed by Dental Practitioners for Ellis Class 2 Fracture-A Questionnaire-based Survey. The Open Dentistry Journal. 2020 Feb 18;14(1)

[11]. Kumar D, Antony S. Calcified Canal and Negotiation-A Review. Research Journal of Pharmacy and Technology. 2018;11(8):3727-30

[12]. Lim KC. Microleakage of intermediate restorative materials. Journal of Endodontics. 1990 Mar 1;16(3):116-8.

[13]. Marchant WE. Intermediate restorative material: A clean and simple mixing technique with predictable results. Journal of Prosthetic Dentistry. 1984 Nov 1;52(5):764.

[14]. Mozayeni MA, Milani AS, Marvasti LA, Asgary S. Cytotoxicity of calcium enriched mixture cement compared with mineral trioxide aggregate and intermediate restorative material. Australian Endodontic Journal. 2012 Aug;38(2):70-5

[15]. Mullaguri H, Suresh N, Surendran S, Velmurugan N, Chitra S. Role of $\mathrm{pH}$ Changes on Transforming Growth Factor- $\beta 1$ Release and on the Fibrin Architecture of Platelet-rich Fibrin When Layered with Biodentine, Glass Ionomer Cement, and Intermediate Restorative Material. Journal of endodontics. 2016 May 1;42(5):766-70.

[16]. Nallaswamy D, Solete P, Subha M. Comparative study on conventional lecture classes versus flipped class in teaching conservative dentistry and endodontics. International Journal of Research in Pharmaceutical Sciences. 2019 Jan 5;10(1):689-93.

[17]. Noor S. Chlorhexidine: Its properties and effects. Research Journal of Pharmacy and Technology. 2016;9(10):1755-60.

[18]. PradeepKumar AR, Shemesh H, Jothilatha S, Vijayabharathi R, Jayalakshmi S, Kishen A. Diagnosis of Vertical Root Fractures in Restored Endodontically Treated Teeth: A Time-dependent Retrospective Cohort Study. J Endod. 2016 Aug:42(8):1175-80. PubmedPMID: 27339633.

[19]. Rajakumar S, Kavitha R, Revanth MP. A comparative evaluation of the marginal sealing ability and compressive strength of different restorative materials-Type VII glass-ionomer cement, Type IX glass-ionomer cement, and intermediate restorative material: An in-vitro study. SRM Journal of Research in Dental Sciences. 2019 Oct 1;10(4):200.

[20]. Rajendran R, Kunjusankaran RN, Sandhya R, Anilkumar A, Santhosh R, Patil SR. Comparative Evaluation of Remineralizing Potential of a Paste Containing Bioactive Glass and a Topical Cream Containing Casein Phosphopeptide-Amorphous Calcium Phosphate: An in Vitro Study. Pesquisa-
BrasileiraemOdontopediatria e ClínicaIntegrada. 2019;19.

[21]. Ramamoorthi S, Nivedhitha MS, Divyanand MJ. Comparative evaluation of postoperative pain after using endodontic needle and EndoActivator during root canal irrigation: A randomised controlled trial. AustEndod J. 2015 Aug;41(2):78-87. PubmedPMID: 25195661.

[22]. Ramanathan S, Solete P. Cone-beam Computed Tomography Evaluation of Root Canal Preparation using Various Rotary Instruments: An in vitro Study. J Contemp Dent Pract. 2015 Nov 1;16(11):869-72. PubmedPMID: 26718293.

[23]. Ravinthar K. Recent advancements in laminates and veneers in dentistry. Research Journal of Pharmacy and Technology. 2018;11(2):785-7.

[24]. Roux D, Doméjean-Orliaguet S, Saade M. Leakage associated with intermediate restorative material and glass-ionomer cement retrograde fillings: A human and sheep teeth comparison with 2 different aging procedures. Oral Surgery, Oral Medicine, Oral Pathology, Oral Radiology, and Endodontology. 2002 Jan 1;93(1):81-7.

[25]. Rajakeerthi R, Ms N. Natural Product as the Storage medium for an avulsed tooth-A Systematic Review. Cumhuriyet Dental Journal. 2019;22(2):24956.

[26]. Schwartz-Arad D, Yarom N, Lustig JP, Kaffe I. A retrospective radiographic study of root-end surgery with amalgam and intermediate restorative material. Oral Surg Oral Med Oral Pathol Oral RadiolEndod. 2003 Oct;96(4):472-7. PubmedPMID: 14561974

[27]. Shahi S, Samiei M, Rahimi S, Nezami H. In Vitro Comparison of Dye Penetration through Four Temporary Restorative Materials. Iran Endod J. 2010 Spring;5(2):59-63. PubmedPMID: 23130028.

[28]. Siddique R, Sureshbabu NM, Somasundaram J, Jacob B, Selvam D. Qualitative and quantitative analysis of precipitate formation following interaction of chlorhexidine with sodium hypochlorite, neem, and tulsi. J Conserv Dent. 2019 Jan-Feb;22(1):40-47. PubmedPMID: 30820081.

[29]. Singh SV, Nikhil V, Yadav S. Effect of cavity varnish and intermediate restorative material on coronal microleakage in endodontically treated tooth. Indian Journal of Dental Research. 2011 Jan 1;22(1):103.

[30]. Wälivaara DÅ, Abrahamsson P, Isaksson S, Salata LA, Sennerby L, Dahlin C. Periapical tissue response after use of intermediate restorative material, gutta-percha, reinforced zinc oxide cement, and mineral trioxide aggregate as retrograde root-end filling materials: a histologic study in dogs. Journal of oral and maxillofacial surgery. 2012 Sep 1;70(9):2041-7.

[31]. Yoshimine Y, Ono M, Akamine A. In vitro comparison of the biocompatibility of mineral trioxide aggregate, 4META/MMA-TBB resin, and intermediate restorative material as root-end-filling materials. Journal of Endodontics. 2007 Sep 1;33(9):1066-9. 